Pieter O. Depuydt Marcio Soares

\section{Cancer patients with ARDS: survival gains and unanswered questions}

Received: 30 June 2014

Accepted: 1 July 2014

Published online: 15 July 2014

(C) Springer-Verlag Berlin Heidelberg and ESICM 2014

\section{P. O. Depuydt}

Department of Intensive Care Medicine, Ghent University Hospital, De Pintelaan 185, 9000 Gent, Belgium

\section{Soares}

Post-Graduation Program, Instituto Nacional de Câncer, Rio de Janeiro, Brazil

M. Soares (

Department of Clinical Research, D'Or Institute for Research and Education, Rua Diniz Cordeiro, 30-Botafogo, Rio de Janeiro, RJ 22281-100, Brazil

e-mail: marciosoaresms@gmail.com

Tel.: +552138836000

Acute respiratory distress syndrome (ARDS) is a devastating diffuse pulmonary inflammation, triggered by a wide array of direct and indirect pulmonary insults, but frequently leading down the common path of acute respiratory failure (ARF) requiring mechanical ventilation (MV), multiorgan dysfunction, and death despite advanced organ support. While no single therapy with curative potential for ARDS has been identified yet, a multimodality approach, including restrictive ventilator settings to limit additional iatrogenic lung injury, careful patient positioning, and judicious use of muscle relaxants, has however led to gradual but marked improvements in survival over the last two decades [1]. Nevertheless, when short-term death is averted, ARDS very often leaves the patient in a debilitated state from which functional recovery is protracted and often incomplete and long-term post-hospital mortality is disproportionally high [2-4]. Of note, severe comorbidities are prevalent in ARDS patients and further contribute to worsen long-term morbidity and mortality in these patients [4].

Remarkably, in an article published recently in Intensive Care Medicine, Azoulay and colleagues provide a relevant contribution to this field by evaluating a large cohort of patients with malignancies and ARDS admitted to 14 referral cancer centers in France and Belgium over a 22-year period [5]. It is well known that patients with solid and hematological malignancies are vulnerable to respiratory complications caused by the underlying malignancy, toxic effects of chemotherapy and, chiefly, infections. Again, over the two last decades we have witnessed that short- and medium-term survival in cancer patients, once ARF occurs, has evolved from dismal to encouraging rates, comparable to (or even surpassing) those of patients with ARF and other major comorbidities. Consequently, the current paper does not come as a total surprise, yet it adds weight to this observation by its large scope in terms of time frame and numbers of patients, its thorough analysis, and by applying the up-to-date Berlin ARDS definitions. As such, it may set a milestone along the winding path of treating critically ill oncological and hematological patients.

There is more to learn from this paper, however. First, it shows that the initial enthusiasm to apply noninvasive ventilation (NIV) as a preferable way to provide mechanical ventilatory support while avoiding the complications of endotracheal intubation in these fragile patients has matured. The sharp rise in NIV use, from 14 to $32 \%$ since the turn of the century, has come to a plateau phase. Moreover, overall NIV failure rates exceeded $70 \%$, particularly in more severely ill patients. In previous studies in cancer patients, ARDS was associated with NIV failure [6-8]. Therefore, the present study provides additional evidence that NIV should not be 
Table 1 Unanswered questions and avenues for research in patients with malignancies and ARDS

How to improve and assist physicians regarding ICU referral and triage criteria for patients with malignancies and ARDS?

What are the efficacy and safety of NIV use in patients with mild to moderate ARDS?

Should preemptive therapy against invasive fungal infections be offered to high-risk patients?

Multidimensional long-term physical, psychological, and cognitive sequelae along with HRQOL in ARDS survivors should be better described and understood

Impact of residual organ dysfunctions and compromise in performance status on the offering (or continuity) of the most appropriate anticancer treatment deserves to be better evaluated

Understanding of the prior two items is essential to assist in the design of rehabilitation programs able to improve the outcomes and HRQOL in ARDS survivors

Post-hospital social and psychological burden in family members and informal caregivers of ARDS survivors should also be assessed

$A R D S$ acute respiratory distress syndrome, ICU intensive care unit, $N I V$ noninvasive ventilation, $H R Q O L$ health-related quality of life

standard first-line support for the oncological/hematological patient with ARDS. In our opinion, until new data from randomized trials become available, NIV can be used cautiously as a supportive tool in some patients with incipient or less severe ARDS, but should be avoided in the moderate and severe Berlin definition categories of ARDS. Second, ARDS is not a single disease, but rather a heterogeneous condition, and reversal or clearance of its trigger is as important as quality of organ support. Such considerations are illustrated again by the divergence in ARDS survival according to its underlying cause. This justifies a thorough diagnostic workup, especially focused on finding a potential infectious etiology. In the study by Azoulay et al. invasive fungal infections, namely pulmonary aspergillosis and Pneumocystis jirovecii pneumonia, accounted for one-third of ARDS cases. Along this line, the effect of preemptive therapy against invasive fungal infections deserves to be evaluated in high-risk patients. Thirdly, severity of illness, as measured by the sequential organ failure assessment (SOFA) at ICU admission, has decreased over time. This could mean that reluctance to call for ICU assistance in case of respiratory deterioration has diminished and that patients are admitted to the ICU in an earlier, still more reversible, phase of their critical illness. This earlier referral policy could have acted as a lever to increase the gains in survival, although evidence to prove this concept remains weak [9].

However, while we may have reached a benchmark to measure future progress, much about ARDS in oncological/hematological patients remains unknown. The full impact of ARDS extends beyond the fact of whether or not patients leave the hospital alive. Within the last few years, several studies have shown that long-term morbidity induced by ARDS is substantial, as patients may suffer from persistent physical constraints and weakness to new-onset neurocognitive disorders, such as depression and long-lasting cognitive impairment [2, 3, 10, 11]. Among the major comorbidities, malignant disease has a major physical and psychological burden in itself [12]. Survivors of ARDS thus may face ARDS-induced deterioration of quality of life superimposed upon that of the underlying malignancy. In addition, poor performance status or persisting organ functional deficits post ARDS may postpone or thwart subsequent aggressive antineoplastic therapy, and thus may prematurely end a therapeutic path which was started with initially curative intentions [13]. Finally, ICU admission, especially involving prolonged mechanical ventilation, is a particularly distressing event not only for the patient but also for the caregivers, who may experience a period of emotional roller coaster ride, owing to uncertainty about short-term survival, being followed by a prolonged period of caregiving [14]. As such, carefully judging the survival benefits of prolonged respiratory support in the light of the oncological or hematological prospects of the patient in the longer run, balanced against the burden imposed on patients and relatives, remains an important exercise in the care of these patients. These should certainly be targets to be addressed in future research (Table 1). Meanwhile, close collaboration and careful communication between intensivist and referring oncologist/ hematologist, each adding their own expertise, are paramount to provide the best care for patients and families.

Acknowledgments Dr. Soares is supported in part by research grants from the National Council for Scientific and Technological Development (CNPq) and Fundação Carlos Chagas Filho de Amparo à Pesquisa do Estado do Rio de Janeiro (FAPERJ), Brazil.

\section{Conflicts of interest None}




\section{References}

1. Tonelli AR, Zein J, Adams J, Ioannidis JP (2014) Effects of interventions on survival in acute respiratory distress syndrome: an umbrella review of 159 published randomized trials and 29 meta-analyses. Intensive Care Med 40:769-787

2. Herridge MS, Tansey CM, Matté A, Tomlinson G, Diaz-Granados N, Cooper A, Guest CB, Mazer CD, Mehta S, Stewart TE, Kudlow P, Cook D, Slutsky AS, Cheung AM, Canadian Critical Care Trials Group (2011) Functional disability 5 years after acute respiratory distress syndrome. $\mathrm{N}$ Engl $\mathbf{J}$ Med 364:1293-1304

3. Needham DM, Dinglas VD, Morris PE, Jackson JC, Hough CL, Mendez-Tellez PA, Wozniak AW, Colantuoni E, Ely EW, Rice TW, Hopkins RO, NIH NHLBI ARDS Network (2013) Physical and cognitive performance of patients with acute lung injury 1 year after initial trophic versus full enteral feeding. EDEN trial follow-up. Am J Respir Crit Care Med 188:567-576

4. Wang CY, Calfee CS, Paul DW, Janz DR, May AK, Zhuo H, Bernard GR, Matthay MA, Ware LB, Kangelaris KN (2014) One-year mortality and predictors of death among hospital survivors of acute respiratory distress syndrome. Intensive Care Med 40:388-396

5. Azoulay E, Lemiale V, Mokart D, Pène $F$, Kouatchet A, Perez P, Vincent F, Mayaux J, Benoit D, Bruneel F, Meert AP, Nyunga M, Rabbat A, Darmon M (2014) Acute respiratory distress syndrome in patients with malignancies. Intensive Care Med. doi: 10.1007/s00134-014-3354-0
6. Adda M, Coquet I, Darmon M, Thiery G, Schlemmer B, Azoulay E (2008) Predictors of noninvasive ventilation failure in patients with hematologic malignancy and acute respiratory failure. Crit Care Med 36:2766-2772

7. Depuydt PO, Benoit DD, Roosens CD, Offner FC, Noens LA, Decruyenaere JM (2010) The impact of the initial ventilatory strategy on survival in hematological patients with acute hypoxemic respiratory failure. J Crit Care 25:30-36

8. Azevedo LC, Caruso P, Silva UV, Torelly AP, Silva E, Rezende E, Netto JJ, Piras C, Lobo SM, Knibel MF, Teles JM, Lima RA, Ferreira BS, Friedman G, Rea-Neto A, Dal-Pizzol F, Bozza FA, Salluh JI, Soares M (2014) Outcomes for patients with cancer admitted to the ICU requiring ventilatory support: results from a prospective multicenter study. Chest. doi: 10.1378/chest.13-1870

9. Song JU, Suh GY, Park HY, Lim SY, Han SG, Kang YR, Kwon OJ, Woo S, Jeon K (2012) Early intervention on the outcomes in critically ill cancer patients admitted to intensive care units. Intensive Care Med 38:1505-1513

10. Chiumello D, Taccone P, Berto V, Marino A, Migliara G, Lazzerini M, Gattinoni L (2012) Long-term outcomes in survivors of acute respiratory distress syndrome ventilated in supine or prone position. Intensive Care Med 38:221-229

11. Guérin C, Girbes AR (2014) Improved ICU outcomes in ARDS patients: implication on long-term outcomes. Intensive Care Med 40:448-450
12. Azoulay E, Mokart D, Pène F, Lambert J, Kouatchet A, Mayaux J, Vincent F, Nyunga M, Bruneel F, Laisne LM, Rabbat A, Lebert C, Perez P, Chaize M, Renault A, Meert AP, Benoit D, Hamidfar R, Jourdain M, Darmon M, Schlemmer B, Chevret S, Lemiale V (2013) Outcomes of critically ill patients with hematologic malignancies: prospective multicenter data from France and Belgium-a groupe de recherché respiratoire en réanimation onco-hématologique study. J Clin Oncol 31:2810-2818

13. Soares M, Toffart AC, Timsit JF, Burghi G, Irrazábal C, Pattison N, Tobar E, Almeida BF, Silva UV, Azevedo LC, Rabbat A, Lamer C, Parrot A, Souza-Dantas VC, Wallet F, Blot F, Bourdin G, Piras C, Delemazure J, Durand M, Tejera D, Salluh JI, Azoulay E, for the Lung Cancer in Critical Care (LUCCA) Study Investigators (2014) Intensive Care in Patients with Lung Cancer: A Multinational Study. Ann Oncol. doi: 10.1093/annonc/mdu234

14. Cameron JI, Herridge MS, Tansey CM, McAndrews MP, Cheung AM (2006) Well-being in informal caregivers of survivors of acute respiratory distress syndrome. Crit Care Med 34:81-86 